\title{
DIGITAL IMAGE CORRELATION AND RELIABILITY-BASED METHODS FOR THE DESIGN AND REPAIR OF PRESSURE PIPES THROUGH COMPOSITE SOLUTIONS
}

\section{Abstract}

This work aims to develop an approach for the reliability-based analysis for the design and repair of pressurized pipes by means of composite solutions. To this end, the approach uses a simulation method to estimate the failure probability of the solution based on the Monte Carlo approach and a Polynomial Chaos Expansion surrogate metamodeling strategy. This combination allows us to reduce the computational time required for evaluating the system's probability of failure as well as extracting the Sobol' indices during the sensitivity analysis stage. The uncertainties related with the composite solution were obtained by means of the Digital Image Correlation approach, allowing us to extract the Probabilistic Distribution Functions (PDF) of its main mechanical parameters. This methodology is validated through the design and repair of a pressurized pipe using a carbon fiber solution and roll wrapping technology. The results show the strong potential of the proposed methodology for the safety evaluation of pressurized composite pipes.

Keywords: Digital Image Correlation, Reliability-based methods, Polynomial Chaos Expansion, Carbon fiber, Pressure Pipes

\section{Nomenclature}

DoE Design of Experiments

E Young's Modulus

21 MIG Mean Intensity Gradient of the speckle pattern

22 PDF Probabilistic Distribution Functions

$P_{f} \quad$ Probability of failure

TSFD Tensile Strength Fiber Direction 
Poisson's ratio

\section{Introduction}

There are many applications of pressurization based on liquid storing and gas and oil transportation, e.g. underground steel pipelines are one of the most effective and safest systems for oil and gas transportation over a long distance $[1,2]$. Due to their exposure to environmental agents, metal pipes can corrode, considerably affecting their useful life and thus the economy of the countries [3]. The behaviour of metal pipes is well known, in terms of both mechanical design and lifecycle problems. Furthermore, most of these applications are subject to different regulations and standards, and are frequently revised in the knowledge progress [4].

Step by step, new material solutions have been replacing the traditional steel applications towards composite materials [5]. This is due to their combination of properties providing a high performance index [6][7] [8], and a good long-term performance [9-11]. Therefore, these new materials represent a very interesting solution for high corrosion and mechanical loaded environments, e.g. chemical industry piping. Within this context, the most common material solution is the fiber-reinforced polymer (FRP), mainly by carbon (CFPR) or glass (GFPR) fiber into an epoxy resin matrix [5, 8, 12-14] . Several techniques could be used for manufacturing FRP pipes, $[1,5,9,12,14,15]$. Among them, roll wrapping technology is interesting, since it opens up a new field of application: the reparation of steel pipes [5, 16-18]. The use of this approach for repairing pipes could overcome the main problems of the traditional methods, as well as offering better resisntance and performance [16].

However, these new composite solutions entails difficulties to determine accurately its properties due to the preesnce of heterogeneous properties [19] and their highly directional behaviour [20] [21]. Within this context, the contact techniques are not capable of properly capturing the mechanical behaviour of these solutions [22-24]. To overcome this drawback, several full-field optical methods have been developed [25][26][27]. Among them, Digital Image Correlation 
(DIC) has become as one of the most promising tools. This method allows us to obtain a full-field of displacements and strains through the use of correlation-based matching procedures and numerical differentiation algorithms [28]. Thanks to this major advantage, DIC has been widely used for the experimental testing of composite solutions under different loading configurations [22][29-31][32-34]. Data obtained by this method is commonly used for the evaluation of strain distribution, damage analysis and microstructure observation [22, 29, 34, 35] and, to a lesser extent, as input for probabilistic analysis [36]. This approach, probabilistic analysis, is considered to be the most reliable strategy for numerical simulation of composite solutions [37] [38, 39]. In contrast to deterministic methods, the main goal of these approaches is to determine the probability of failure of a mechanical system under the influence of different uncertainties, such as loads or material properties among others [38]. Studies carried out by Rafiee and Ali Torabi [38] and Rafiee et al. [40] [39], highlights the relevance of considering these manufacturing uncertainities since it is highly likely that composite prototypes experience faliures bellow the deterministic value. As stated Sriramula and Chryssanthopoulos [21], these uncertainities mght be considered at a constitutent, ply or component level. The estimation of this probability of failure could be carried out by means of approximation methods such as the First (FORM) and Second Order Reliability (SORM) methods or even by means of simulation strategies such as the Monte Carlo Sampling (MCS) method [41]. MCS highlights for its simple and direct implementation [38, 39]. However, the cost of this approach increases rapidly when a low probability of failure is required [37], requiring a huge number of simulations and a large computational time [38]. To cope with this lamination, variance reduction techniques and metamodels are commonly used [37][42]. As a result of these techniques, it is possible to construct an equivalent and computationally inexpensive mathematical replica of the system, allowing the carrying out of huge numbers simulations with great accuracy.

As a consequence, this paper therefore aims to progress the effective integration of the DIC approach with the latest advances in probabilistic analysis of engineering solutions. To this end, 
obtaining the failure probability of a composite solution used for the design and repair of pressurized pipes. In the Methods (Section 2) we describe the composite materials used as well as the experimental and numerical strategies adopted. In Section 3, we show the experimental results obtained by the combination of DIC and the reliability approach in the design and repair of pressurized pipes. Finally, in the Conclusion (Section 4) we summarise the findings and discuss future studies.

\section{Materials and methods}

\subsection{Composite solution evaluated}

Compared to other materials or other composites solutions such as GFPR, carbon fiber capabilities exceed those of its competitors. It should be noted that the surface of the carbon fiber is a microcrystalline graphite structure, which entails that the distribution of stresses and the generation and propagation of fissures can be adjustable [43]. In addition, its resistance to high temperatures and corrosion must be considered. All this makes carbon fiber the most suitable material for high-performance pipes [44].

Hence, the material used for testing was a carbon fiber polymer reinforcement CC $200 \mathrm{~T}-120{ }^{\circledR}$ with a thickness of $0.32 \mathrm{~mm}$ (Table 1);Error! No se encuentra el origen de la referencia., an epoxy resin matrix CR82® and a hardener Biresin CH80-10®. Technical characteristics of epoxy resin and hardener are the same as used by García-Martin et al. [36]. The material has been made from a $200 \mathrm{~g}$ Plain where 9 plates of $21 \times 29 \mathrm{~cm}$ have been obtained. The mix ratio, for a specific amount of $200 \mathrm{~g}$, was $158 \mathrm{~g}$ of resin plus $42 \mathrm{~g}$ of hardener.

Table 1: Technical characteristics of fibers CC 200T-120®.

\begin{tabular}{cc}
\hline Mechanical properties & Values \\
\hline Thickness (mm) & 0.32 \\
\hline Density (warp x weft) (end/cm) & 1.76 \\
\hline
\end{tabular}




\begin{tabular}{cc}
\hline Tex (warp x weft) & $500 \times 500$ \\
\hline Width (cm) & $120+/-1$ \\
\hline Weight $(\mathrm{g} / \mathrm{m} 2)$ & $200+/-5$ \\
\hline Weight per roll $(\mathrm{kg})$ & 110 \\
\hline Tensile strength (MPa) & 3530 \\
\hline & 1.5 \\
\hline
\end{tabular}

99

100 The first layer of reinforcement was placed with proper orientation, then it was impregnated by

101 the resin and catalyst mixture. This process was repeated a total of nine times giving as a result a

102 composite solution made up by nine layers: $[ \pm 90] 9$. Each time a layer was added, a light pressure

103 was exerted in order to remove the resin surplus. To obtain the desired thickness of $2 \mathrm{~mm}$ [45],

104 the mold was machined with the proper measure. The block was cured in a drying oven for 50

105 minutes, at a temperature of $50^{\circ} \mathrm{C}$. Once the material was prepared, the specimens were machined

106 with the proper dimensions (Figure 1). During this process, computer numerical controlled cutting

107 was used, and programmed according to current regulations specifications [45].

108

109

110 a)

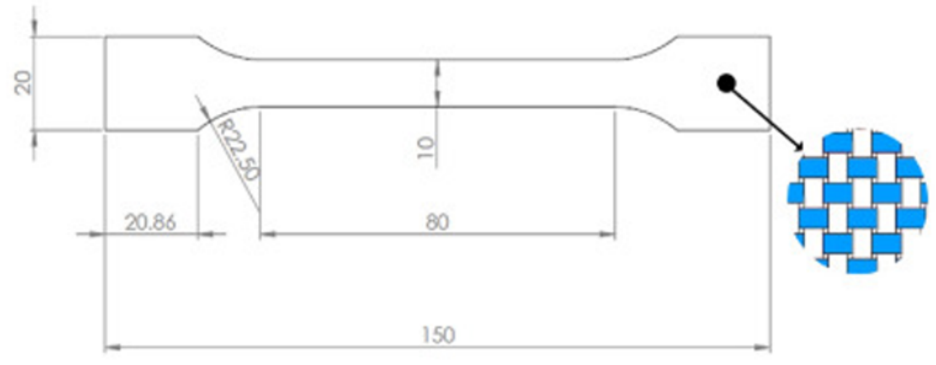

b)

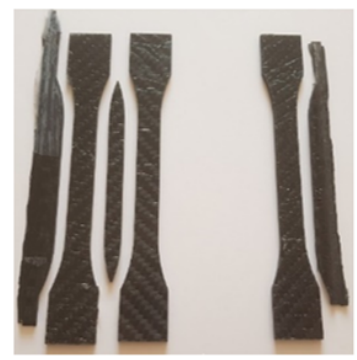

Figure 1: Manufacturing process: a) dimensions of the tested specimens in mm and; b) image of the specimens. 

correlation method

115 The composite solution was evaluated by means of tensile tests according to guideline ISO 527

116 [45]. The electromechanical tensile machine Servosis ME-405/50/5 technical specifications are 117 shown in Table 2.

Table 2: Servosis ME-405/50/5 technical specifications.

\begin{tabular}{cc}
\hline Maximum Load & $500 \mathrm{kN}$ \\
\hline Load Cell & REP Transducer Type TC4 50kN \\
\hline Grip & MTS Model XSA304A \\
\hline
\end{tabular}

119

$132\left(u, v, \frac{\delta u}{\delta x}, \frac{\delta u}{\delta y}, \frac{\delta v}{\delta x}, \frac{\delta v}{\delta y}\right)^{T}$. the load cell.
To capture the displacement and strains suffered by the composite solution during the tensile tests a 2D-DIC approach was used. The acquisition of these images was carried out by means of the DIC prototype developed by García-Martin et al. [36]. This prototype is composed of a high resolution camera Canon EOS 700D with a $60 \mathrm{~mm}$ prime macro-lens and a PLC connected with

Once the images were acquired, a standard DIC protocol was applied in order to obtain the full field of displacements and strains. During this stage, the Region Of Interest (ROI) of each image was split into subsets [46] which allow the images to be tracked through the Zero mean Normalized Cross-Correlation (ZNCC) index due to its robustness [47]. During this tracking stage, it was assumed that each subset could suffer a deformation result of the linear combination of six degrees of freedom (translation, elongation and shear deformation in the $\mathrm{x}$ and $\mathrm{y}$ axis) (Figure 2) (Eq. 1-2) for which the basic form of displacement of a subset is

$$
x^{\prime}=u+\frac{\delta u}{\delta x} \Delta x+\frac{\delta u}{\delta y} \Delta y
$$




$$
y^{\prime}=v+\frac{\delta v}{\delta x} \Delta x+\frac{\delta v}{\delta y} \Delta y
$$

133 where $x^{\prime}$ and $y^{\prime}$ are the final displacements of the subset; $u$ and $v$ are the displacements

134 components of the subset center $P ; \Delta x$ and $\Delta y$ are the distance between the initial center's subset

135 and the final position and; $\frac{\delta u}{\delta x}, \frac{\delta u}{\delta y}, \frac{\delta v}{\delta x}, \frac{\delta v}{\delta y}$ are the displacement gradients of the subset.

a)

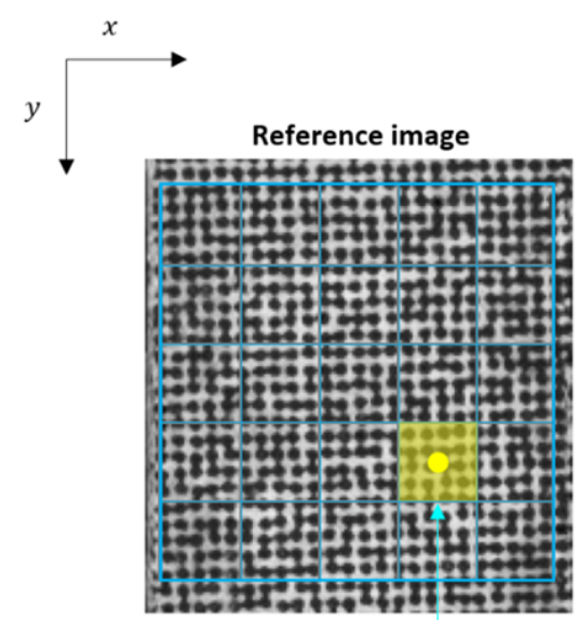

Reference subset

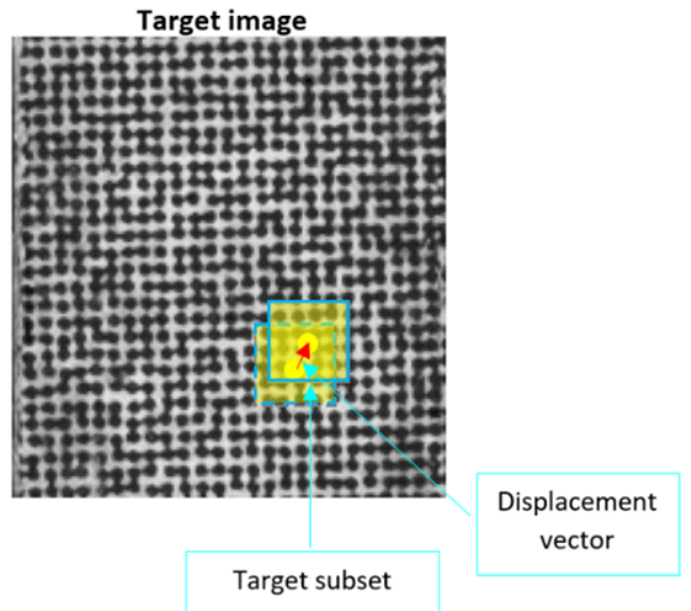

b)

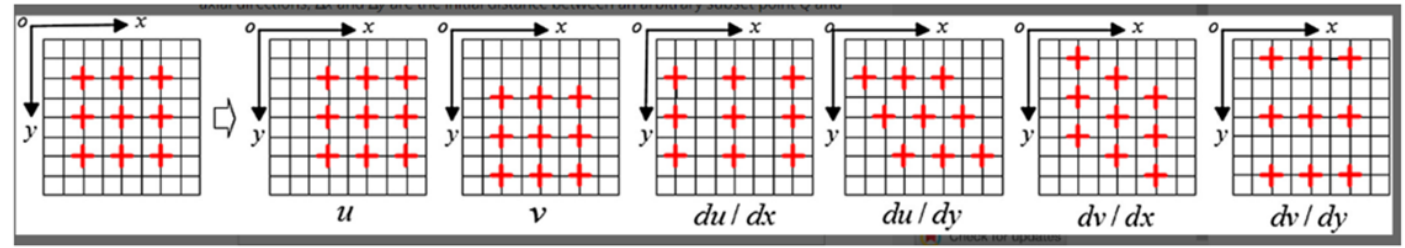

136

Figure 2: Graphical representation of the DIC approach: (a) evaluation of the displacement suffered by a subset and (b) degrees of freedom considered during the displacement analysis.

139 Complementary to the ZNCC index, and with the aim of obtaining sub-pixel accuracy, a

140 refinement stage was carried out by means of the following strategies: i) a bi-quantic b-spline

141 interpolation scheme to pass from the discrete values of the images (0-255) to a continuum space

142 and; ii) the Inverse Composition Gauss-Newton method for the minimization of the cost function 
that match the reference subset with the deformed one. For more details about the algorithms used

144 the reader is referred to [47].

145 The procedure previously shown was reproduced in all the image's subsets allowing us to obtain

146 a full-field of displacements. The strains of the specimens were calculated by means of the Green-

147 Lagrangian strain tensor using the gradients obtained during the evaluation of the displacements

148 [36]. In order to obtain the full field strain, this procedure was applied throughout ROI.

149 The success of the tracking carried out during the DIC approach strongly depends on the random intensity distribution of the ROI, wich must present the following features: distinct, unique, nonperiodic and stable grayscale [48]. Due to the absence of a proper gray variation on the specimen's surface, it was necessary to apply an artificial Speckle pattern using the approach defined by Garcia-Martin et al. [36]. This strategy allows us to obtain a suitable Speckle pattern through the perturbation of a regular circular grid, wich was printed and applied with the help of an elastic prime over the specimen surface.

Apart from the considerations previously shown, the images captured by the sensor suffer from lens distortion that could be considered as another source of error [47]. The minimization of this error requires the removal of the these radial and tangential distortions. Therefore, the present case applied the calibration procedure proposed by Vo et al. [49].

In order to guarantee the accuracy and quality of the data obtained with the DIC approach, it is mandatory that all previously determined steps are carried out by skilled technicians. First, the prototype must be properly configured to synchronize all data. A bad Speckle pattern with insufficient contrast could cause the subsets not to be detected correctly and the correlation was not performed. In addition, all parameters have to be defined according to the test configuration for the correlation algorithm runs properly. Finally, the calibration process has to be carefully 
carried out in order to obtain a high accuracy in the results, since a bad calibration could cause

167 incoherent displacements and strains.

168

169

182

183

184

\subsection{Numerical strategy for the reliability analysis}

As stated in the Introduction, the main goal of the reliability analysis is to find the probability function of a mechanical system under the influence of different uncertainties. These uncertainties could be considered at material (random variables and random field) or ply level (layer-wise random variable). For the present study, we considered the mechanical uncertainties of the composite solution at ply level. These uncertainties were extracted by means of the DIC approach previously described. The evaluation of the probability function was carried out through the Monte Carlo (MC) approach following the next formulation (Eq. 3). The input data required for the MC simulation was obtained combining a finite element modelling simulation with a surrogate metamodeling strategy, allowing reduction of computational costs as well as the estimation of the Sobol' indices for the sensitivity analysis of the mechanical solution. The proposed workflow was carried out with different number of plies until the probability of failure of the model was less than the minimum threshold of probability of failure (optimal solution) (Figure 3). This failure threshold corresponds to the reliability target $(\beta)$ which guarantees the safety of the model.

$$
P_{f}=\operatorname{Prob}[G(Y) \leq 0]=\int_{G(Y) \leq 0} p_{y}(Y) d Y
$$

where $P_{f}$ is the probability of failure; $G$ is the performance function; $Y$ is the variable's vector and $p_{y}$ is the joint probability density function. 


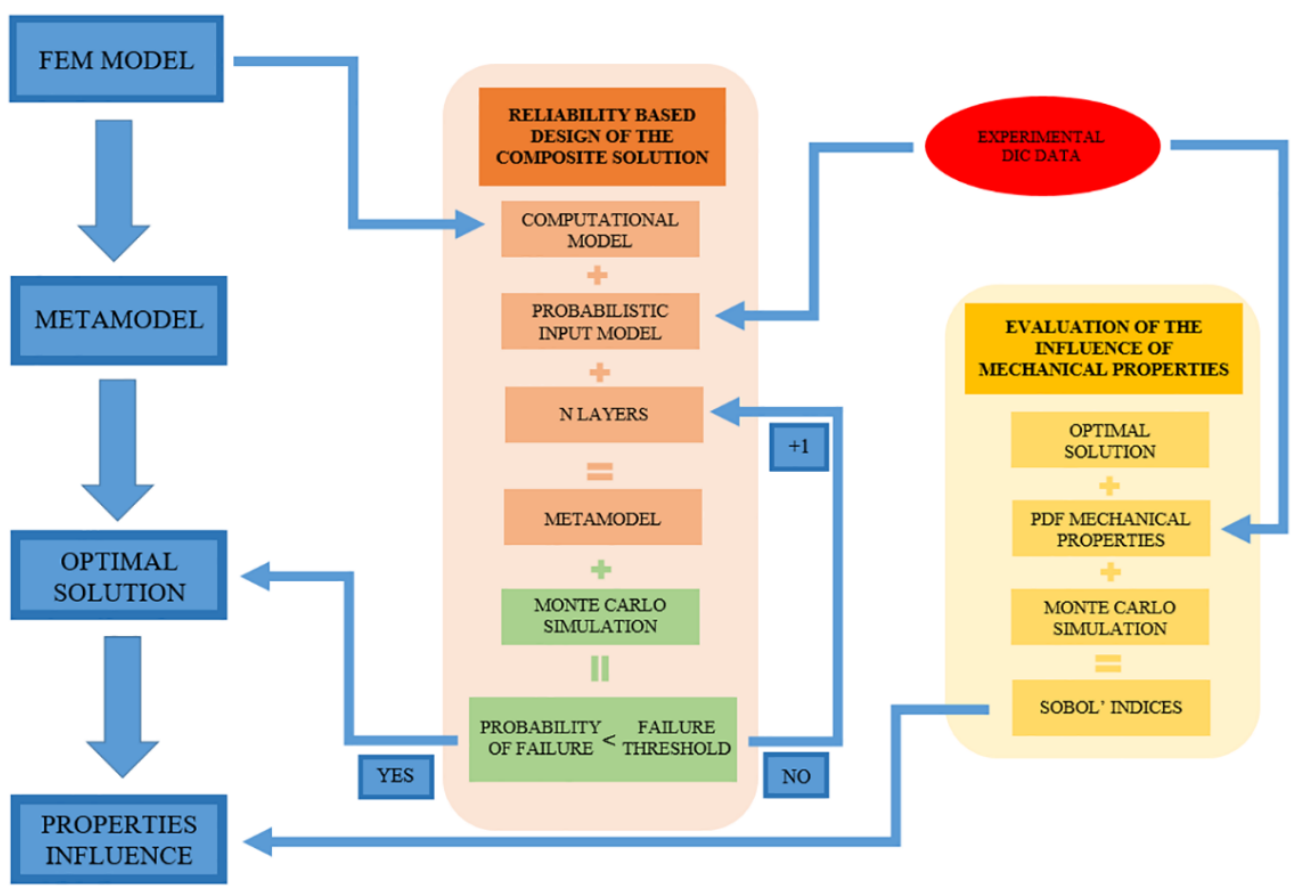

Figure 3: Workflow adopted for the reliability analysis.

2.3.1 Definition of the performance function for the reliability analysis: the Tsai-Wu failure criterion

190 Among the different methods able to simulate the failure of composite solutions, the Tsai-Wu

191 failure criterion is one of the most used in the literature $[7,12,14,50]$. This criterion assumes that

192 the failure of a composite solution takes places when the Failure Index (FI) (Eq. 4) is higher than

$1931[51]$.

$$
\begin{aligned}
& F I=F_{1} \sigma_{1}+F_{2} \sigma_{2}+F_{3} \sigma_{3}+F_{11} \sigma_{1}^{2}+F_{22} \sigma_{2}^{2}+F_{33} \sigma_{3}^{2}+2 F_{12} \sigma_{1} \sigma_{2}+2 F_{23} \sigma_{2} \sigma_{3}+ \\
& +2 F_{31} \sigma_{3} \sigma_{1}+F_{44} \sigma_{4}^{2}+F_{55} \sigma_{5}^{2}+F_{66} \sigma_{6}^{2} \leq 1
\end{aligned}
$$

where:

$$
\begin{array}{ccc}
F_{1}=\frac{1}{X_{T}-\frac{1}{X_{C}}} & F_{2}=\frac{1}{Y_{T}-\frac{1}{Y_{C}}} & F_{3}=\frac{1}{Z_{T}-\frac{1}{Z_{C}}} \\
F_{11}=\frac{1}{X_{T} X_{C}} & F_{22}=\frac{1}{Y_{T} Y_{C}} & F_{33}=\frac{1}{Z_{T} Z_{C}} \\
F_{44}=\frac{1}{S_{y z}^{2}} & F_{55}=\frac{1}{S_{z x}^{2}} & F_{66}=\frac{1}{S_{x y}^{2}} \\
F_{12}=\left(-\frac{1}{2}\right) \sqrt{F_{11} F_{22}} & F_{23}=\left(-\frac{1}{2}\right) \sqrt{F_{22} F_{33}} & F_{31}=\left(-\frac{1}{2}\right) \sqrt{F_{33} F_{11}}
\end{array}
$$


$Y_{T} \equiv$ Tensile strength in the transversal isotropic surface

$Z_{T} \equiv$ Tensile strength in the transversal isotropic surface

$Y_{C} \equiv$ Compressive strength in the transversal isotropic surface

$Z_{C} \equiv$ Compressive strength in the transversal isotropic surface

$S_{x y} ; S_{y z} ; S_{z x} \equiv$ Shear strength in the transversal isotropic surface

According to the formulation previously exposed, the performance function of a composite solution in terms of Tsai-Wu failure criterion could be expressed as (Eq. 5). Taking this into consideration, the composite solution is operating in the safety range if $G>0$ and in the failure state if $G<0$, and the surface $G=0$ is the limit state of the solution.

$$
G=1-F I
$$
where $G$ is the performance function and $F I$ is the failure index obtained from the Tsai-Wu criterion.

\subsubsection{Metamodeling strategy}

210 The proposed methodology considers the use of the MC sampling method for solving the

211 probability of failure of the system (Eq. 3). To achieve reliable results, thousands of simulations

212 are required for solving the problem. The computational cost of this task is unacceptable most of 213 the times, so it is necessary to establish an alternative that offers reliable results with a lower computational cost. In this context, the so-called surrogate models or metamodels allow the Input-

215 Output response of a complex system to be approximated with a low number of inputs thanks to 216 its compactness and analytical scalability. Within this context, one of the most robust and used 
217 metamodeling strategies is the Polynomial Chaos Expansion (PCE) [52], especially in the 218 propagation of uncertainties in engineering applications [53].

219 Initially developed by Wiener [54], the PCE is a stochastic metamodeling strategy that approximates the behaviour of a system by means of a spectral representation of random variables in terms of a set of multivariate polynomials. This method assumes that the physical model, in this case the numerical simulation, can be represented as a finite variance model, $f(X)$, whose input $x$ is a random vector of independent and constrained variables $X \epsilon R^{M}$. The independence of the input parameters allows us to build these polynomials as a tensorization of univariate polynomials with respect to the marginal PDF's and where the mathematical equation can be summarized as follows (Eq. 6).

$$
Y \approx f(X)=\sum_{\alpha \in A} \gamma_{a} \varphi_{a}(X)
$$

where $\alpha=\left\{\alpha_{1} \cdots \alpha_{M}\right\}$ is the multi-index, $A \subset N^{M}$ is a set of indices for the multivariate orthonormal polynomials, $\gamma_{a}$ are deterministic coefficients to be computed and $\varphi_{a}(X)$ are multivariate orthonormal polynomials.

The estimation of the coefficients exposed in (Eq. 6) was carried out by means of a least square minimization problem between the vector of random inputs $(X)$ and the model responses $(Y)$ as

232 follows (Eq. 7):

$$
\varphi_{\alpha}=\operatorname{argmin} \frac{1}{N} \sum_{i=1}^{N}\left[\gamma^{(i)}-\sum_{\alpha \in A} \gamma_{\alpha} \varphi_{a}\left(x^{(i)}\right)\right]^{2}
$$

233 In order to overcome a possible over-fitting situation in the presence of high-dimensional inputs,

234 the proposed methodology uses the adaptive sparse PCE based on the least angle regression 235 proposed by Blatman and Sudret [55]. This method applies the least angle regression algorithm 

strategy proves to be very effective in engineering problems with high dimensions [55].

It is worth mentioning that all the metamodels are built from a limited number of inputs coming from the Design of Experiments (DoE). Thus, it is essential to assess the quality of the computed surrogate model. To this end, we propose to be use the modified version of the Leave-one-out error [57] (Eq. 8-9). This metric of error offers a good compromise between fair error estimation and affordable computational cost.

$$
\begin{gathered}
\text { LOO error }=\frac{1}{N} \sum_{i=1}^{N}\left(\frac{Y\left(X^{(i)}-f^{\sim P C E}\left(X^{i}\right)\right)}{1-h_{i}}\right)^{2} \\
\text { LOOerror }^{*}=\text { LOOerror } *\left(1-\frac{\operatorname{cardA}}{N}\right)^{-1}\left(1+\operatorname{tr}\left(\varphi^{T} \varphi\right)^{-1}\right)
\end{gathered}
$$
where $Y\left(X^{(i)}\right)$ is the computational model; $f^{\sim P C E}\left(X^{(i)}\right)$ is the surrogate model obtained from a specific DoE with $N$ samples; $h_{i}$ is the i-th diagonal term of matrix $A\left(A^{T} A\right)^{-1} A^{T} ; A$ the experimental matrix; card $A$ is the number of terms in the truncate series and; $\varphi=$ $\left\{\varphi_{i j}=\varphi_{j}\left(x^{(i)}\right), i=1, \ldots, N ; j=1, \ldots, \operatorname{card} A\right\}$.

The reduced computational cost of the surrogate metamodeling strategy allows the application of the MCS for different purposes. According to this, it is possible to calculate the influence of each input in the final result of the model. Within this context one of the most used strategies is the estimation of the Sobol' indices. These indices assume that the variance of the model (output) can be described as a sum of the variances of the inputs (Eq. 10). The normalized version of each variance with respect to the total one allows us to obtain de Sobol' indices with different orders (from 1 to $2^{\mathrm{n}-1}$ ) (Eq. 11). The sum of these indices is the total Sobol' index whose value is equal to 1 .

$$
V(Y)=\sum_{i} V_{i}+\sum_{i} \sum_{j>i} V_{i j}+\sum_{i} \sum_{j>i} \sum_{k>j} V_{i j k}+\ldots V_{123 . . N}
$$


where $V(Y)$ is the variance of the model; $V_{i}=V\left(E\left(Y \mid X_{i}\right)\right)$ is the first order partial variance; $V_{i j}=V\left(E\left(Y \mid X_{i}, X_{j}\right)\right)$ is the second order partial variance, etc.

$$
S_{i}=\frac{V_{i}}{V(Y)}, S_{i j}=\frac{V_{i j}}{V(Y)}, \text { etc }
$$

where $S_{i}$ is the first order Sobol' index and $S_{i j}$ is the second-order Sobol' indices.

\section{Experimental results}

\subsection{Analysis of the roll wrapping solution by means of 2D-DIC}

\subsubsection{Test setup}

A total of fifty-one tensile tests were carried out following the BS EN ISO 527-5:2009 guidelines [45]. The images for the DIC analysis were adquired each $400 \mathrm{~N}$ of traction force increment, taking the first image without load in order to obtain the reference image. Previously to the tests, a preparation stage was carried out with the aim of optimizing the results obtained during the tensile tests. This stage comprised the following steps: i) definition of the ground sample distance (GSD) as well as the aperture of the lens; ii) application of the Speckle pattern and; iii) the geometrical calibration of the camera.

The images were acquired with the same camera configuration used by García-Martin et al. [36]. Taking these values into account, a Speckle pattern was designed using the methodology proposed in Section 2.2, using a diameter of $0.324 \mathrm{~mm}$ and a step of $0.432 \mathrm{~mm}$ and obtaining a covering factor of $44 \%$ [58]. Finally, this pattern was printed and applied on the specimen surfaces by means of an elastic prime (Figure 4). The quality of the pattern was evaluated through the Mean Intensity Gradient (MIG) value [59], obtaining an average value of 50. This value was considered acceptable taking into account the method used [59].

277 For the camera calibration we used the calibration approach defined in Section 2.2, adquiring a total of 22 images. The following inner parameters were obtained (Table 3). 


\begin{tabular}{|c|c|c|c|}
\hline \multicolumn{2}{|c|}{ Parameter } & \multirow{2}{*}{$\begin{array}{c}\text { Initial } \\
1.36286 \times 10^{4}\end{array}$} & \multirow{2}{*}{$\begin{array}{c}\text { Refined } \\
1.5206 \times 10^{4}\end{array}$} \\
\hline & $f_{u}$ & & \\
\hline & $f_{v}$ & $1.36286 \times 10^{4}$ & $1.5208 \times 10^{4}$ \\
\hline \multirow{2}{*}{ Principal point ${ }^{*}$} & $u$ & $7.2084 \times 10^{2}$ & $2.5118 \times 10^{3}$ \\
\hline & $v$ & $1.3378 \times 10^{3}$ & $2.0986 \times 10^{3}$ \\
\hline \multirow{3}{*}{$\begin{array}{l}\text { Radial distortion } \\
\text { coefficients }\end{array}$} & $a_{0}$ & 0 & $-8.5316 \times 10^{-2}$ \\
\hline & $a_{1}$ & 0 & $3.3055 \times 10^{-1}$ \\
\hline & $a_{2}$ & 0 & $1.0130 \times 10^{1}$ \\
\hline \multirow{2}{*}{$\begin{array}{l}\text { Tangential distortion } \\
\text { coefficients }\end{array}$} & $p_{0}$ & 0 & $-3.0724 \times 10^{-3}$ \\
\hline & $p_{1}$ & 0 & $1.5447 \times 10^{-2}$ \\
\hline
\end{tabular}

280

281

a)

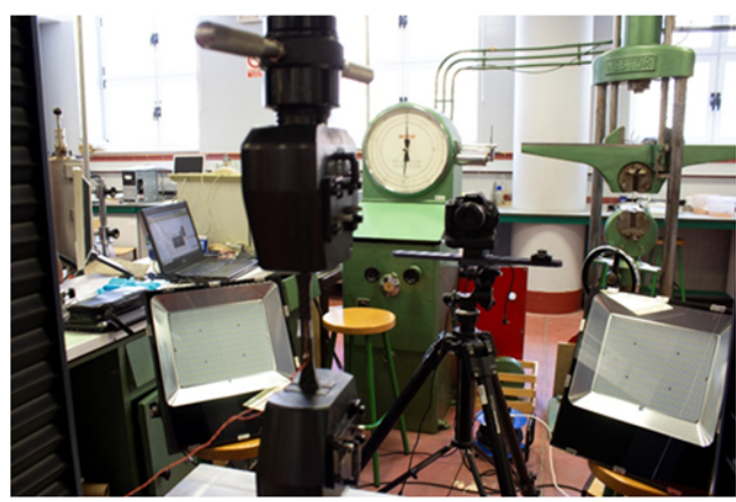

b)

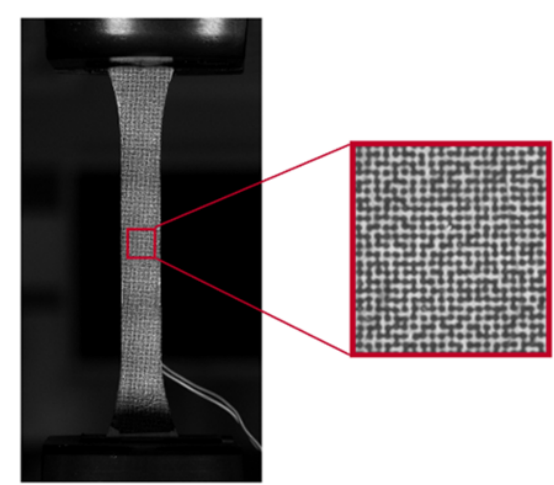

Figure 4: Digital Image Correlation campaign: a) set-up used and; b) detail of the Speckle pattern applied.

\subsubsection{Displacement and strain results}

In order to obtain the displacamet and strain on each test specimen, DIC approach defined in Section 2.2 was carried out with the open-source software Ncorr [60]. A subset size of $20 \times 20$ pixels and a $35 \%$ overlap (step of 7 pixels) were considered to ensure a proper DIC configuration [46], obtaining a full fiel displacement and strains in all the ROI (Figure 5). 

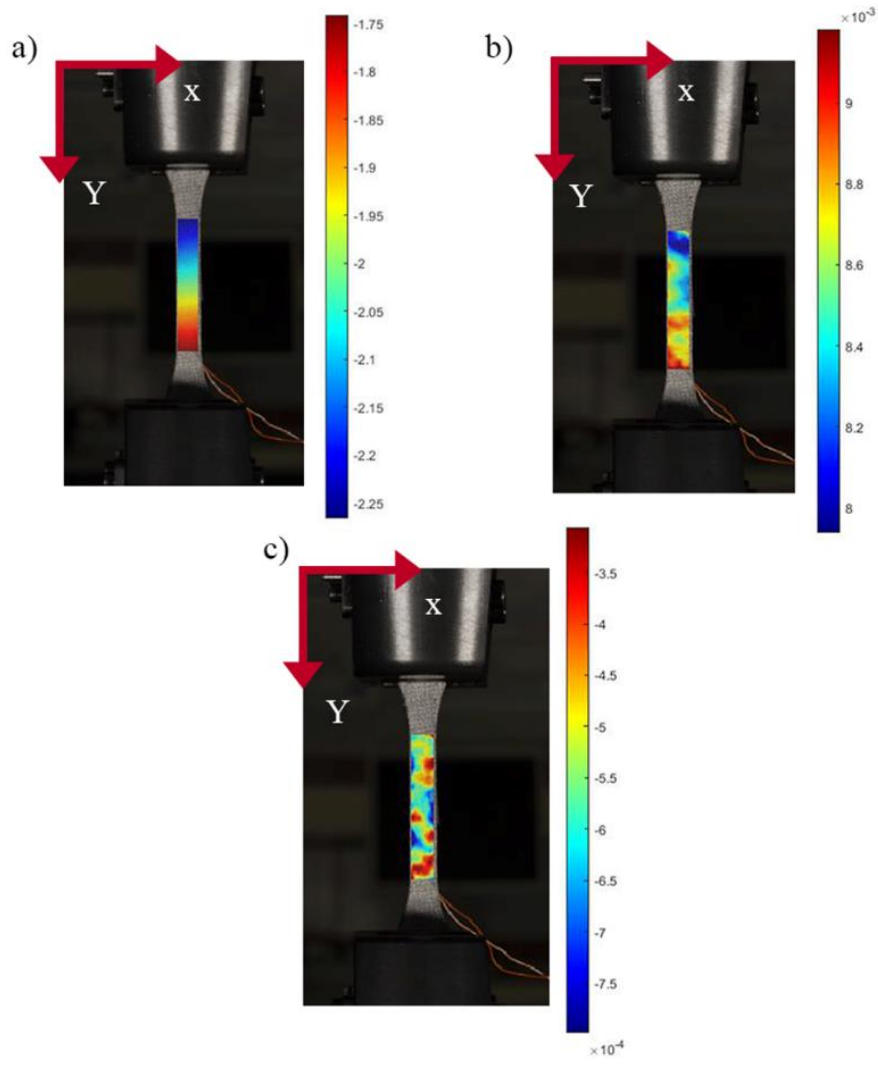

291

292 Figure 5: Displacements and strains obtained by the 2D-DIC approach: a) displacements (Y) in mm; b) strains $(Y)$ in $\mathrm{mm} / \mathrm{mm}$ and c) strains $(X)$ in $\mathrm{mm} / \mathrm{mm}$.

294 Taking into consideration the test set-up, the state of stresses along the central area (ROI area) could be considered to be constant. Therefore, if the material is homogeneous, the expected maximum principal strains will be reached at the same time in all the ROI. However, the results of the DIC tests revealed a heterogeneous distribution of the strains in the longitudinal and transverse directions and thus the presence of different mechanical properties along the composite structure. These heterogeneities could be attributed to local variations during the manufacturing process [36].

\subsubsection{Extraction of the PDF functions}

As stated in Section 2.3, the reliability analysis requires prior knowledge of the probability density functions of the material's variables. The population of each variable evaluated by means of the DIC approach was extracted during the next strategy (Figure 6)(Table 4): i) creation of several 

maximum principal strain for the evaluation of the ultimate capacity of the material.

307 With the aim of obtaining a wide population, eight virtual extensometers were placed in each specimen. Four of them were placed vertically ( $y$ axis) and other four were placed horizontally ( $x$ axis) (Figure 6). In this way, a total of 204 values were obtained for the Young's Modulus and Poisson's ratio.

311 This wide population allowed to calculate a significant average value and covariance associated

312 with each of the parameters. In this sense, it is worth mentioning the high value of the covariance associated with the Poisson's ratio. Generally high covariance values of the Poisson's Ratio are obtained in comparison with those of other parameters [61] [36]. These high values are mainly

315 due to the importance of fiber alignment in the manufacturing process as well as the low average value of the Poisson's ratio of the CRFP.

317 The reliability analysis approach proposed in Section 2.3 allow quantifying the uncertainty of the mechanical properties previously obtained. In order to introduce this uncertainty, it was necessary to adjust these data to a probabilistic distribution.

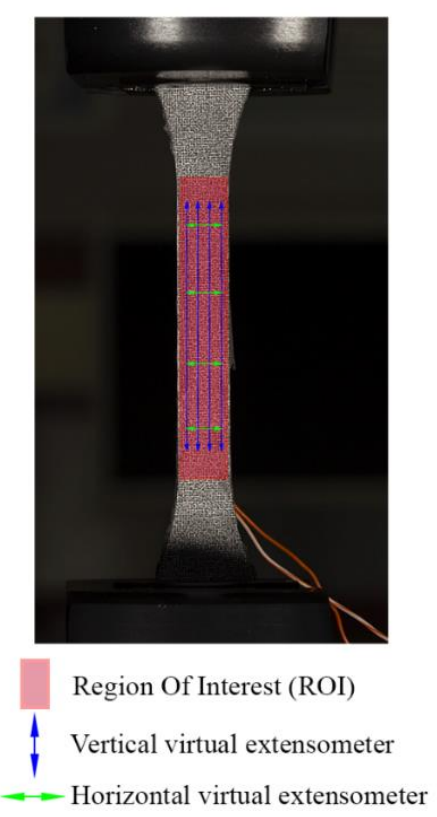


Figure 6: Extraction of the mechanical parameters: evaluation of the Young's modulus and Poisson's ratio by means of virtual extensometer.

323 Table 4: Results obtained from the mechanical characterization of the specimens using DIC approach.

\section{Composite solution evaluated}

\begin{tabular}{cccccc}
\hline Parameter & $\begin{array}{c}\text { Number of } \\
\text { data }\end{array}$ & Mean & $\begin{array}{c}\text { Covariance } \\
(\%)\end{array}$ & $\begin{array}{c}\text { Lower } \\
\text { bound }\end{array}$ & $\begin{array}{c}\text { Upper } \\
\text { bound }\end{array}$ \\
\hline$E$-Young's modulus (GPa) & 204 & 50.7088 & 4.07 & 45.0583 & 57.0596 \\
\hline$v$-Poisson's ratio (-) & 204 & 0.0574 & 41.95 & 0.0138 & 0.1462 \\
\hline $\begin{array}{c}T \text {-Maximum principal tensile } \\
(\mathrm{MPa})\end{array}$ & 51 & 402.5128 & 8.33 & 309.5652 & 461.5762 \\
\hline
\end{tabular}

Along with this test, the goodness-of-fit tests of Chi-Square, Kolmogorov-Smirnov and Anderson-Darling were performed. These techniques allow us to accept or reject the hypothesis that mechanical properties are drawn from populations with a specified distribution [62]. Finally, a curve fit method was performed with the aim of obtaining the PDFs.

The candidate PDFs considered were Normal, Log-Normal, Weibull, and Gamma. The acceptance/rejection of distribution is provided in Table 5 where zero indicates acceptance of assumed distribution parameters, and unity indicates rejection. As shown in Table 5, for some parameters there are several candidate PDFs which pass all tests.

Table 5: Goodness of fit (GOF) probability; N-Normal, LN-Lognormal, W-Weibull, G-Gamma; 0Accept and 1-Reject. Chi-Square (Chi), Kolmogorov-Smirnov (KS) and Anderson-Darling (AD) tests of the Young's Modulus $(E)$, Poisson's ratio $(v)$ and Maximum principal tensile $(T)$.

\begin{tabular}{cccccccccccccc}
\hline PDF & & N & \multicolumn{1}{c}{ LN } & & & W & \multicolumn{3}{c}{ G } \\
\hline GOF & Chi & KS & AD & Chi & KS & AD & Chi & KS & AD & Chi & KS & AD \\
\hline$E$ & 1 & 0 & 0 & 0 & 0 & 0 & 1 & 1 & 1 & 0 & 0 & 0 \\
\hline$v$ & 1 & 0 & 1 & 0 & 0 & 0 & 1 & 0 & 0 & 0 & 0 & 0 \\
\hline$T$ & 0 & 0 & 0 & 0 & 0 & 0 & 0 & 0 & 0 & 0 & 0 & 0 \\
\hline
\end{tabular}


337 Along with these statistical tools, the physical considerations of the parameters were taken into 338 account, obtaining a Log-Normal distribution for the Young's Modulus and Poisson's Ratio and 339 a Weibull distribution for the maximum principal tensile stress (Table 6 and Figure 7). These 340 curves are consistent with those obtained in similar experiments [61].

Table 6: Probabilistic distribution functions of the Young's Modulus $(E)$, Poisson's ratio $(v)$ and Maximum principal tensile $(T)$ of the specimens.

\begin{tabular}{cccc}
\hline Parameter & Distribution & $\begin{array}{c}\mu \text { (Log-Normal)/ } \\
\text { A (Weibull) }\end{array}$ & $\begin{array}{c}\sigma \text { (Log-Normal)/ } \\
\text { B (Weibull) }\end{array}$ \\
\hline$E$ & Log-Normal & 3.9253 & 0.0405 \\
\hline$v$ & Log-Normal & -2.9422 & 0.4176 \\
\hline$T$ & Weibull & 417.0948 & 14.8402 \\
\hline
\end{tabular}
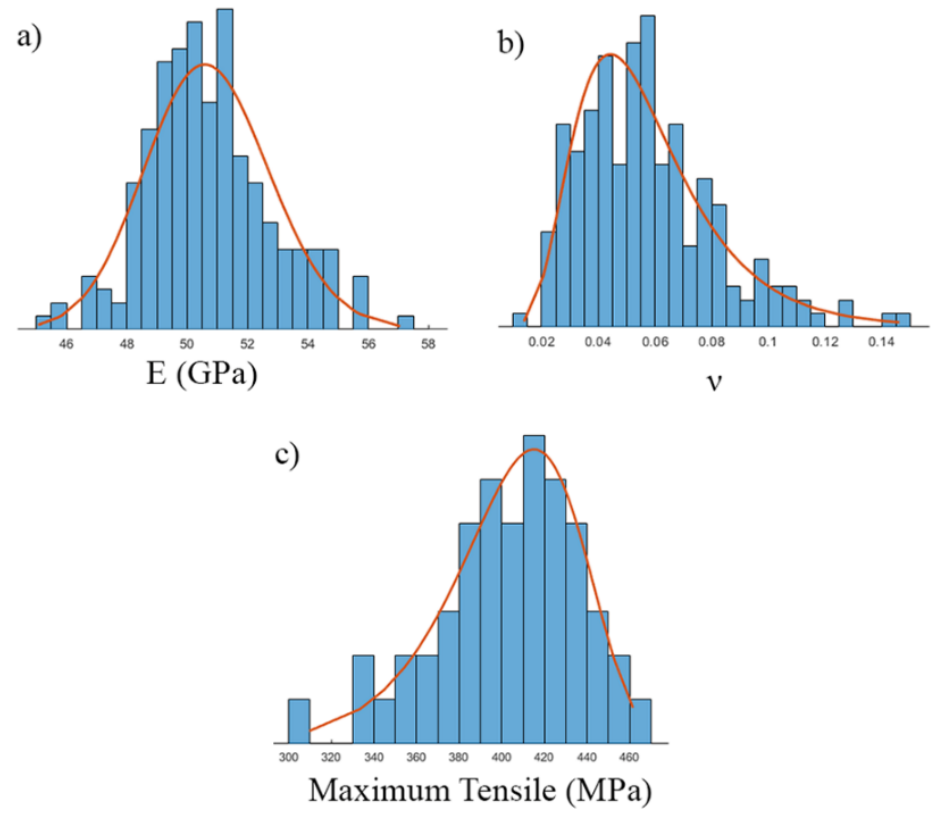

Figure 7: Graphical representation of the PDFs of each parameter: (a) Log-Normal distribution of the Young's modulus (E); (b) Log-Normal distribution of the Poisson's ratio ( $v$ ) and (c) Weibull distribution of the maximum principal tensile $(T)$. 
349 Taking into account the main applications of the roll-wrapping technique, two numerical models were considered for the reliability analysis: i) the design of a new composite pipe and; ii) the repair of a corroded steel pipe. Both numerical simulations were carried out in the FEM software ABAQUS 2019®.

On the one hand, the composite pipe was modelled considering the following parts: i) a nonstructural polymer liner with $2 \mathrm{~mm}$ of thickness; ii) a composite wrap made up of several composite layers with $0.32 \mathrm{~mm}$ of thickness. The wrap part was meshed as a continuum shell with enormously the time needed to run the models. This is a paramount aspect to consider because the models had to be repeated 200 times in order to obtain the surrogate models, as it was explained in Section 2.3.2. So, convergence studies were performed using elements between 20 and $1 \mathrm{~mm}$ size. It was observed that using elements smaller than $10 \mathrm{~mm}$ the results did not improved significantly, but the sizes of the whole models and the time to run them grew exponentially. Finally, a $10 \mathrm{~mm}$ size element was considered enough precise for both models. For the loading and boundary conditions, we used an internal pressure on the inside wall of the pipe of 11.2 MPa and plane strain boundary constraints. Under these conditions the principal stresses which works in the pipe were the hoop tensile stresses [63].

On the other hand, we also considered a corroded steel pipe retrofitted with the composite solution exposed in Section 2.1. and by means of roll-wrapping technology. In this sense, the numerical model was made up of the following parts: i) a steel pipe with $4 \mathrm{~mm}$ of thickness; ii) a corroded area on which the effective thickness is $2 \mathrm{~mm}$ (half of the initial one); iii) a non-structural putty layer and; iv) a roll-wrapping solution made up of different composite layers of $0.32 \mathrm{~mm}$ thickness 

previously exposed.

The number of layers in each numerical simulation were determined during the reliability analysis. To this end, the iterative approach defined in Section 2.3. was applied, defining a maximum probability of failure of 0.00135 (reliability index of 3.0). This strategy was carried out in the mathematical software MATLAB with the assistance of the open-source library UQLab [64].

a)

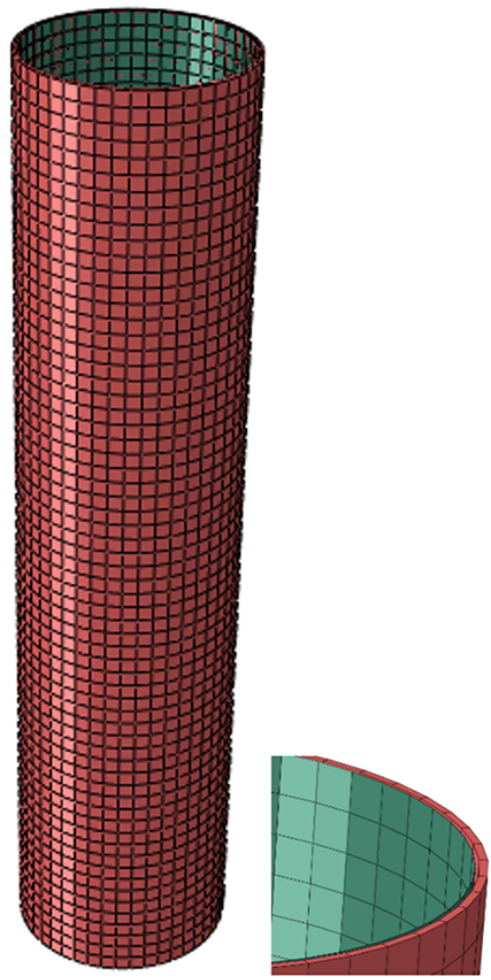

b)

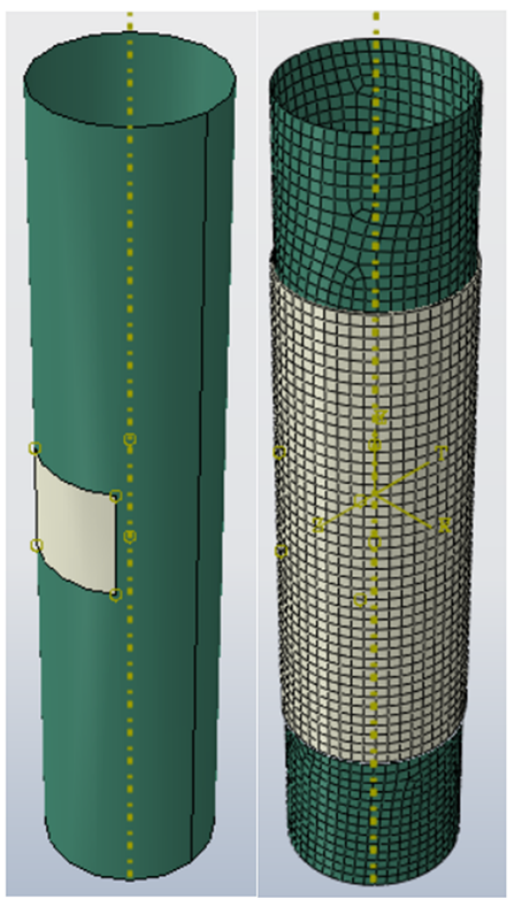

Figure 8: Numerical meshes used: a) new composite steel pipe and; b) retrofitting of a corroded steel pipe.

\subsection{Results obtained during the design of the new composite pipe}

With the aim of obtaining the optimal number of layers for the composite pipe previously shown, an iterative reliability analysis proposed in Section 2.3. was carried out. During each iteration a PCE of the model response was built considering as random inputs the mechanical variables of each layer namely: i) Young's Modulus; ii) Poisson's ratio and; iii) tensile strength. The PDFs of 
these variables were those obtained in the DIC evaluation (Figure 7). Throughout this process we used the Latin Hypercube Sampling method to create the DoE [65]. This technique is a popular method that allows us to obtain a random DoE ensuring the uniformity of each sample in the domains of the inputs. From the present study case several sizes of the DoE were used to build the metamodel. The accuracy of the metamodel was evaluated by means of the LOO error exposed in (Eq. 8-9). The metric of this error was complemented by a cross correlation on which we used a different DoE with the same size. The sparsity of the metamodel was carried out by varying the maximum degree of the polynomials from 1 to 20 , where the optimal degree was selected according to the smallest LOO error.

After a total of 15 iterations the reliability analysis found a solution which satisfied the failure criteria. In this situation the degree of the polynomials was 5 with an associated LOO error of 0.0047 and a cross-validation error of 0.005 .

According to the numerical results obtained, it was necessary to use a total of 15 CFPR layers to satisfy the failure criteria previously defined $\left(P_{f}<0.00135, \beta>3\right)$ (Table 7 and Figure 9$)$. In this situation, the solution has a probability of failure of 0.00108 and a reliability index of 3.0645 (Figure 10).

Table 7: Results of probability of failure $\left(P_{f}\right)$ and reliability index $(\beta)$ obtained during the reliability analysis of the composite pipe with $10,11,12,13,14$ and 15 layers.

\begin{tabular}{ccc}
\hline Number of layers & $\boldsymbol{P}_{\boldsymbol{f}}$ & $\boldsymbol{\beta}$ \\
\hline $\mathbf{1 0}$ & 0.5061 & 0.0154 \\
\hline $\mathbf{1 1}$ & 0.1661 & 0.9696 \\
\hline $\mathbf{1 2}$ & 0.0480 & 1.6645 \\
\hline $\mathbf{1 3}$ & 0.0132 & 2.2200 \\
\hline $\mathbf{1 4}$ & 0.00356 & 2.6912 \\
\hline $\mathbf{1 5}$ & 0.00108 & 3.0645 \\
\hline
\end{tabular}


408

409

410

411

412 In order to understand which mechanical variables are the most relevant in the safe design of the

413 composite pipe, a Sobol sensitivity analysis was carried out. To this end a new MCS was

414 performed, using in this context a total of 500,000 simulations. During this process it was assumed

415 that all the layers have the same mechanical properties according to the PDF obtained during the

416 DIC analysis (Figure 7). The figure indicates that the tensile strength of the material is the most

417 relevant mechanical parameter in the safety of the solution followed by the Young's Modulus. a)

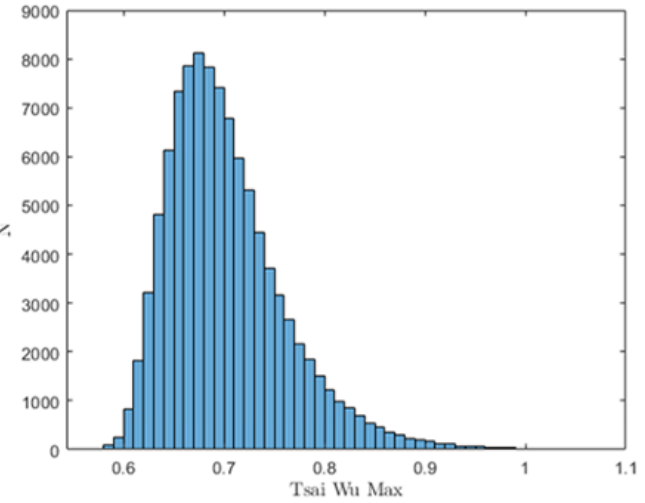

Figure 9: Convergence of the $P_{f}$ and $\beta$ during the Monte Carlo Simulation. b)

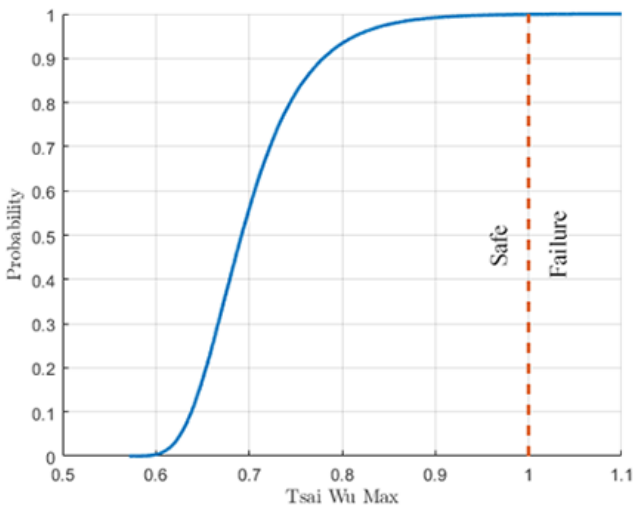

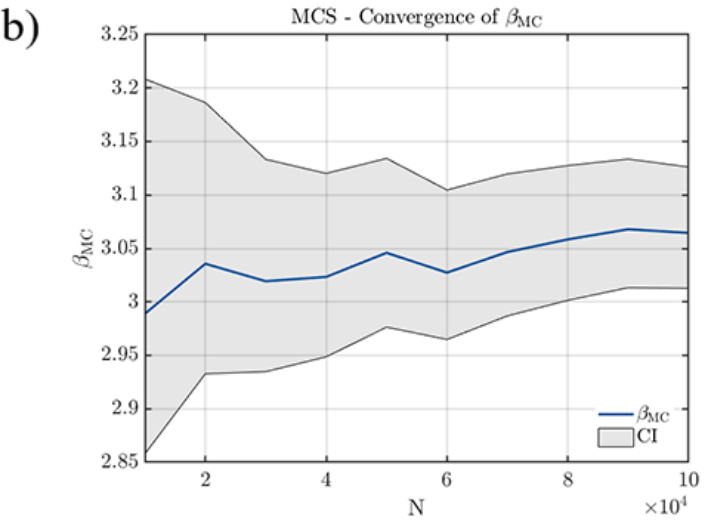

b) a)

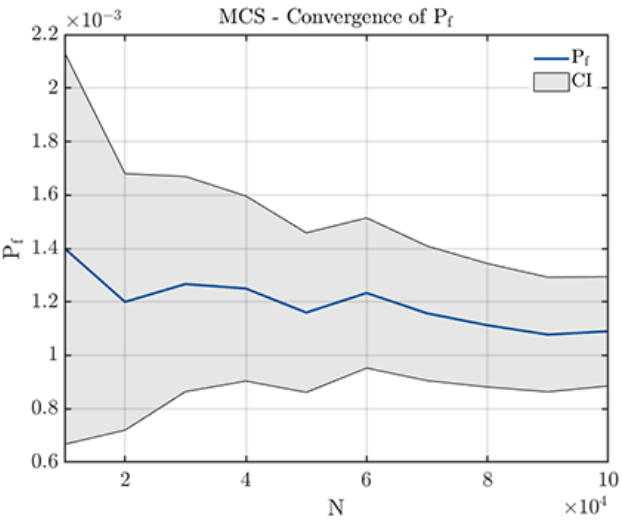

.


418 This mechanical property explains $89.00 \%$ of the total variance (Figure 11 ). The great similarity

419 between the first and the total Sobol-indices highlights the absence of a second-order effect.

420 a)

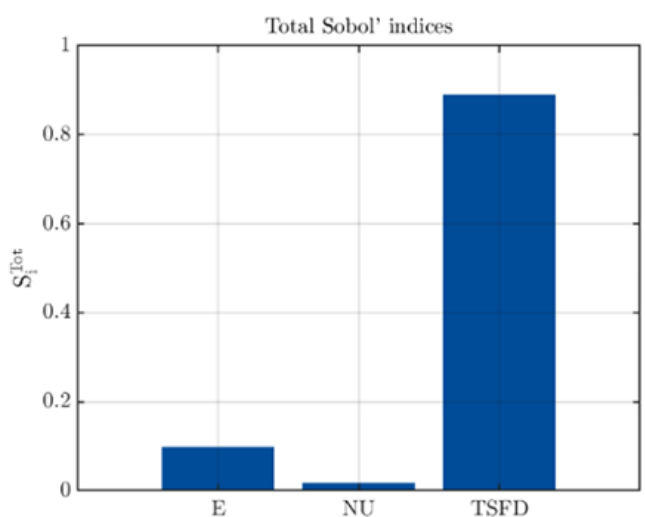

b)

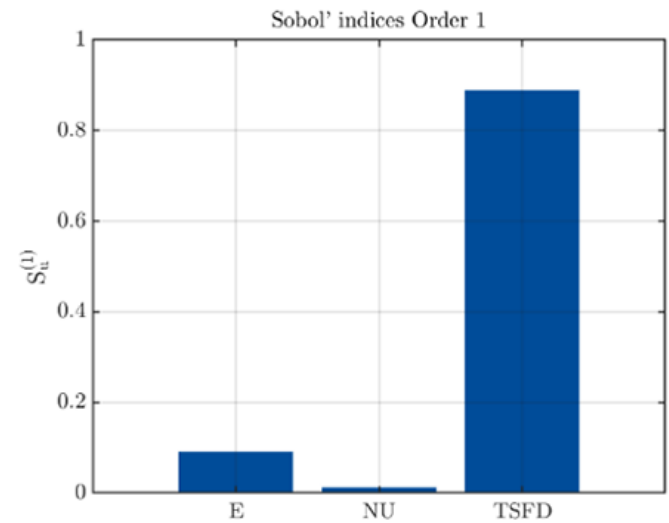

Figure 11: Results obtained during the sensitivity analysis: a) Total Sobol' indices and; b) First-order Sobol' indices.

\subsection{Reliability evaluation of a corroded steel pipe retrofitted with CFPR}

Apart from the design of new composite pipes, another relevant application of the method could involve the retrofitting of existing steel pipes. Within this context the most common damage is the corrosion of the pipe which reduces the resistance section of it. According to this, and considering the numerical model of the corroded steel pipe defined in Section 3.2, the proposed iterative reliability analysis was performed. During this analysis we used as inputs the mechanical variables obtained by DIC (Figure 7) as well as the thickness of the corroded area. The PDF of this input was assumed uniform, varying from $0 \%$ (totally corroded) to $100 \%$ (no corrosion).

The results of the iterative reliability analysis showed that the minimum number of layers required to fulfil the safety requirements $\left(P_{f}<0.00135, \beta>3\right)$ are 4 . With this set-up the probability of failure is $1 \times 10^{-5}$ and the reliability index is 4.26 , using MCS with $10^{5}$ simulation (Figure 12). These simulations were obtained by means of a PCE metamodel with maximum polynomial degree of 

resulting from the cross-validation estimated in 0.003 .

a)

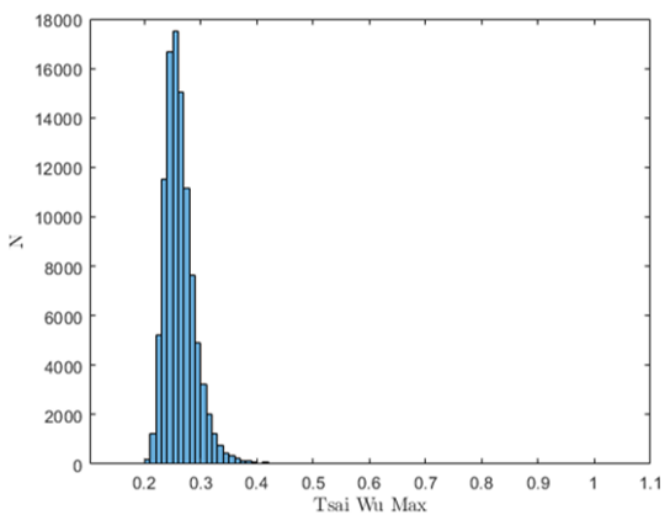

c)

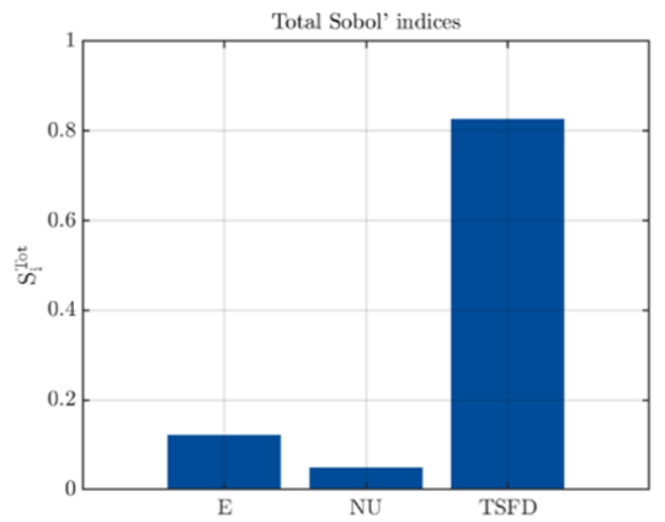

b)

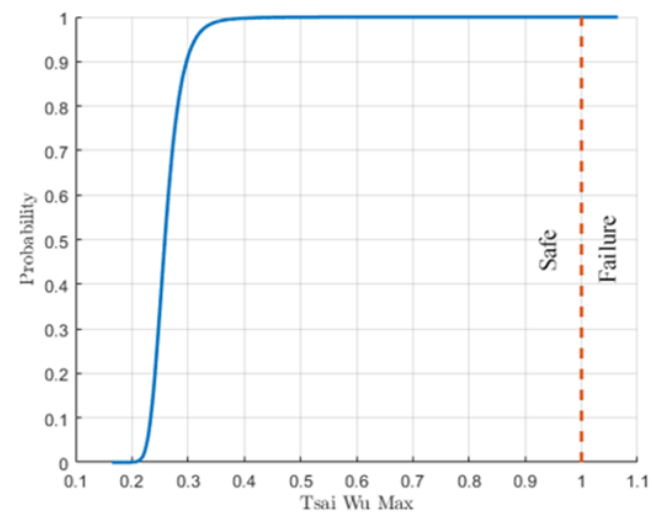

d)

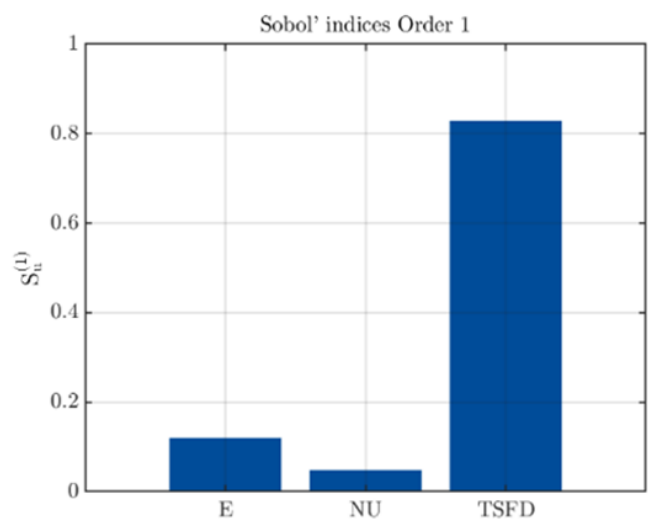

437

438

439

440

441

442

443

444

Figure 12: Results obtained during the reliability analysis of the corroded pipe retrofitted with 4 layers of CFPR: a), b) PDF and CFD functions and; c), d) Sobol' indices.

It is worth highlighting that the thickness of the corroded part is a critical parameter in this type of reliability analysis, determining the number of layers required to retrofit the steel pipe. Hence, it is neccessary to know the minimum thickness of steel for which the 4 CFPR layers fulfil the safety requirements. To this end, a constrained optimization procedure was carried out using as cost function the thickness of the corroded part and a probability of failure of 0.00135 , corresponding to a reliability index of 3. In order to achieve this result, the proposed methodology uses the genetic algorithm described by Goldberg [66]. In this procedure the process starts with an initial population which is repeatedly modified by randomly selecting individuals from the current population and using them as parents to produce the offspring of the next generation. During this process a crossover method creates new individuals on which a mutation strategy 
ensure that a few genes are modified, exploring a new search space. After several successive

451 generations, the population "evolves" towards an optimal solution [67]. For the present study case

452 the genetic algorithm was based on a population size of 25 individuals with a maximum number

453 of generations equal to 75 . For the sequence of creating new populations one individual was

454 chosen to automatically pass to the next generation. The fraction of offspring was established in

$45575 \%$. In order to obtain the global minimum of the problem, a total of 3 runs were carried out,

456 selecting the run with the best fitness value (Figure 13). The results of this optimization show that

457 the critical effective thickness is $0.61 \mathrm{~mm}$ ( $15 \%$ of the initial section). In this situation the 4-layer

458 CFPR solution has a reliability index of 3.01 (Figure 14 and 15).

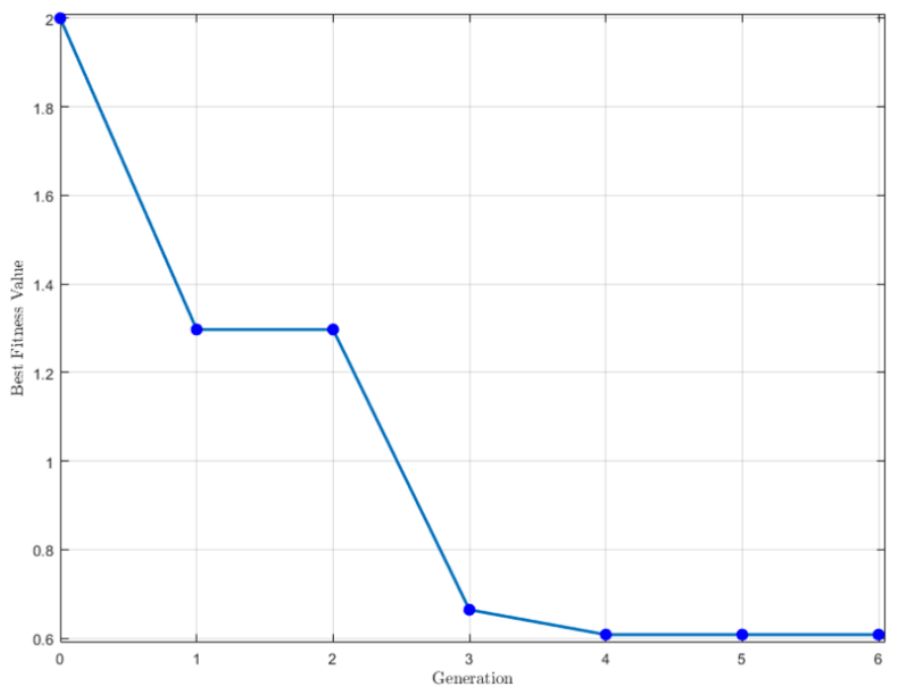

Figure 13: Convergence plot obtained during the minimization stage.

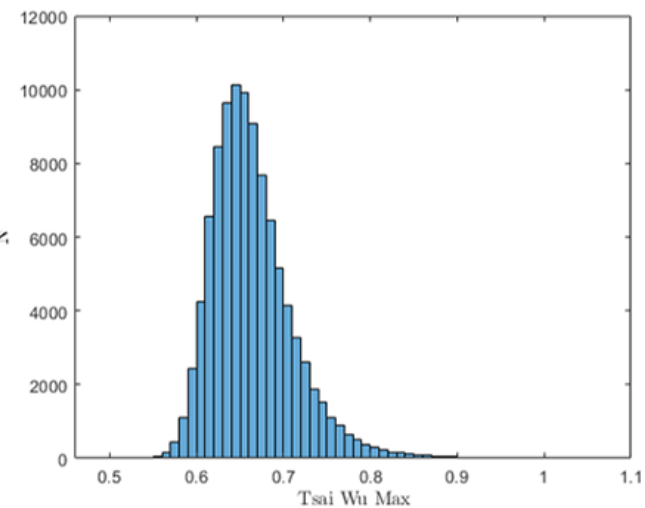

b)

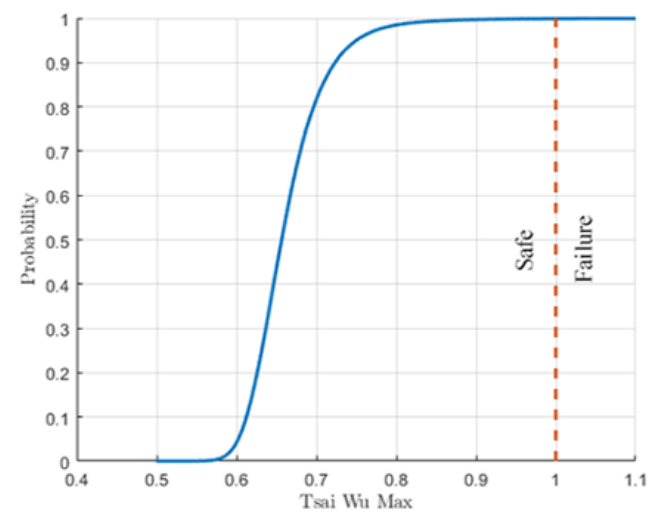


Figure 14: Results obtained during the reliability analysis of a corroded pipe (15\% of the initial section) retrofitted with 4 layers of CFPR: a) PDF and; b) CFD functions.

a)

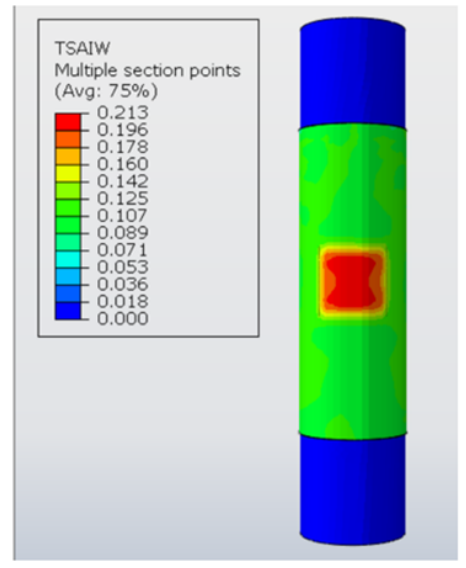

b)

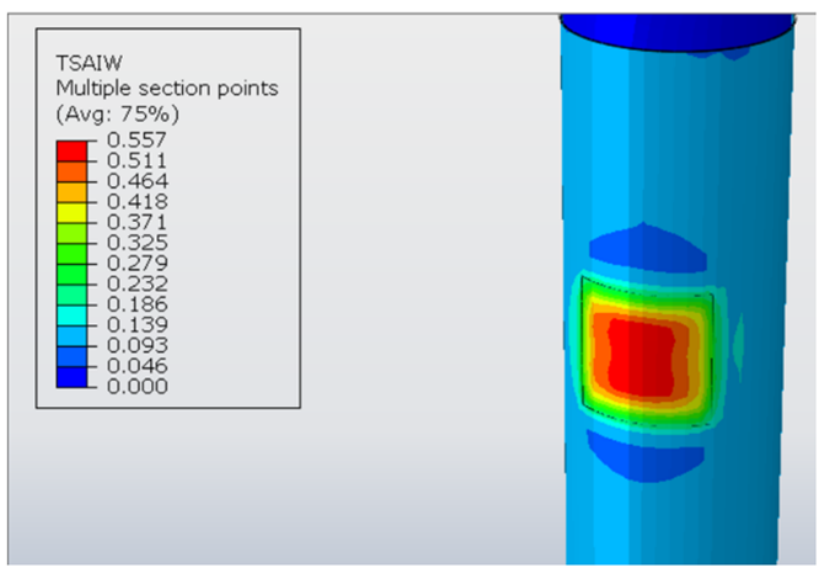

Figure 15: Distribution of the Tsai-Wu values along the composite solution: a) in a steel pipe with 50\% of corrosion and; b) in a steel pipe with $85 \%$ of corrosion (maximum value for which the 4-layer CFPR solution is reliable).

\section{Conclusions}

In this paper a new methodology for the reliability analysis of pressurized composite pipes was presented. To this end the method uses the data provided by the digital image correlation approach for the generation of probabilistic distribution function of the different mechanical properties of the composite solution. We subsequently use this data to build a surrogate model of numerical simulation, taking into account the stochastic nature of this type of solution. Therefore, an Adaptive Sparse Polynomial Chaos Expansion was used where the accuracy is evaluated by means of the Leave one Out error. On the one hand, the Digital Image Correlation approach offers a robust and low-cost alternative to traditional contact measurement techniques such as linear variable differential transformers or electrical resistance strain gauges. This alternative is especially attractive in probabilistic approaches for which a large number of inputs are required. In this situation it is possible to exploit the full-field nature of the data by means of virtual extensometers that allowus to extract the mechanical properties of a sample from different locations. On the other hand, the use of an Adaptive Sparse Polynomial Chaos Expansion allows us to mimic the response of a numerical model with great efficiency, requiring a relative low number of inputs to obtain a good Leave one Out error. For example, the two numerical models considered during the present study requires only 200 samples to build an accurate metamodel 
with an average Leave one Out Error of 0.005 . This metric of error, which is compared with a standard cross validation, is especially efficient since it does not require extra samples to be 491 obtained for the validation. Once the metamodel is obtained, the cost of each evaluation is extremely low, allowing us to carry out a reliability analysis, or even a sensitivity evaluation, by means of the Monte Carlo method. In the context of this study, one numerical evaluation by means of the FEM method requires 25 secs and assumes the investment of 29 days to carry out one Monte Carlo simulation. In contrast, the use of PCE metamodeling requires only 2 hours to carry out this simulation $(0.3 \%$ of the time required to perform this evaluation with FEM simulations alone). Additionally, this surrogate model can be used for optimization purposes (e.g. evaluation of the minimum effective thickness that is able to support a specific composite solution). In this situation the efficient computation cost of the surrogate model allow us to use evolutionary optimization strategies able to find the global optimal of the solution as well as the use of inequalities that introduces uncertainty in the safety evaluation. Within this context the system was able to save $99.7 \%$ of the total time required to obtain the optimal solution by means of FEM simulations. All these simulations were carried out in a Intel® XEON E3-1240 v3 processor running at $3.4 \mathrm{GHz}$ with $8 \mathrm{~GB}$ RAM DDRII.

Future works will be focused on the use of full-field data provided by the DIC approach to evaluate the uncertainties of the composite solution from a material level by means of Random Field theory. Additionally, the proposed methodology will be used within the context of reliability-based design optimization, integrating minimization algorithms that allow us to estimate the optimal disposition of the composite solution. Also, the proposed method will be applied to other fabrics (plain /satin weave) and materials, where different reinforcements (GDPR) and matrix (especially thermoplastic ones) will be tested. 
514 [1] Sinclair G, Helms J. A review of simple formulae for elastic hoop stresses in 515 cylindrical and spherical pressure vessels: What can be used when. International Journal 516 of Pressure Vessels and Piping. 2015;128:1-7.

517 [2] Yahaya N, Noor NM, Othman SR, Sing LK, Din MM. New technique for studying 518 soil-corrosion of underground pipeline. Journal of Applied Sciences. 2011;11:1510-8.

519 [3] Koch GH, Brongers MP, Thompson NG, Virmani YP, Payer JH. Corrosion cost and 520 preventive strategies in the United States. United States. Federal Highway 521 Administration; 2002.

522 [4] Veritas DDN. DNV-OS-C501-Composite Components-Offshore Standard. 2003.

523 [5] Mokhtari M, Nia AA. The application of CFRP to strengthen buried steel pipelines 524 against subsurface explosion. Soil Dynamics and Earthquake Engineering. 2016;87:5252562.

526 [6] Ashby MF. Chapter 5 - Materials Selection. Materials selection in mechanical design.: 527 Kidlington. Oxford, UK: Butterworth-Heinemann, cop; 2011. p. 97-124.

528 [7] Rafiee R. On the mechanical performance of glass-fibre-reinforced thermosetting529 resin pipes: A review. Composite Structures. 2016;143:151-64.

530 [8] Laney P. Use of composite pipe materials in the transportation of natural gas. Idaho 531 International Engineering and Environmental Laboratory, Bechtel BWXT Idaho, LLC. 5322002.

533 [9] Rafiee R, Mazhari B. Evaluating long-term performance of Glass Fiber Reinforced 534 Plastic pipes subjected to internal pressure. Construction and Building Materials. 535 2016;122:694-701.

536 [10] Rafiee R, Mazhari B. Simulation of the long-term hydrostatic tests on glass fiber 537 reinforced plastic pipes. Composite Structures. 2016;136:56-63.

538 [11] Rafiee R, Elasmi F. Theoretical modeling of fatigue phenomenon in composite pipes. 539 Composite Structures. 2017;161:256-63.

540 [12] Velosa JC, Nunes JP, Antunes P, Silva J, Marques A. Development of a new 541 generation of filament wound composite pressure cylinders. Composites Science and 542 Technology. 2009;69:1348-53.

543 [13] Zaman A, Gutub SA, Wafa MA. A review on FRP composites applications and 544 durability concerns in the construction sector. Journal of Reinforced Plastics and 545 Composites. 2013;32:1966-88.

546 [14] Colombo C, Vergani L. Optimization of filament winding parameters for the design 547 of a composite pipe. Composites Part B: Engineering. 2018;148:207-16

548 [15] Rafiee R, Torabi MA, Maleki S. Investigating structural failure of a filament-wound 549 composite tube subjected to internal pressure: experimental and theoretical evaluation. 550 Polymer Testing. 2018;67:322-30.

551 [16] Lim KS, Azraai S, Noor N, Yahaya N. An overview of corroded pipe repair techniques using composite materials. Int J Mater Metall Eng. 2016;10:19-25.

[17] Mahdi E, Eltai E. Development of cost-effective composite repair system for oil/gas pipelines. Composite Structures. 2018;202:802-6.

[18] Kara M, Uyaner M, Avci A. Repairing impact damaged fiber reinforced composite pipes by external wrapping with composite patches. Composite Structures. 2015;123:18.

[19] Rafiee R, Fakoor M, Hesamsadat H. The influence of production inconsistencies on the functional failure of GRP pipes. Steel and Composite Structures. 2015;19:1369-79.

560 [20] Reddy JN. Mechanics of laminated composite plates and shells: theory and analysis:

561 CRC press; 2003. 
[21] Sriramula S, Chryssanthopoulos MK. Quantification of uncertainty modelling in 563 stochastic analysis of FRP composites. Composites Part A: Applied Science and Manufacturing. 2009;40:1673-84 [22] Orell O, Vuorinen J, Jokinen J, Kettunen H, Hytönen P, Turunen J, et al. Characterization of elastic constants of anisotropic composites in compression using digital image correlation. Composite Structures. 2018;185:176-85.

[23] Sharifi S, Gohari S, Sharifiteshnizi M, Alebrahim R, Burvill C, Yahya Y, et al. Fracture of laminated woven GFRP composite pressure vessels under combined lowvelocity impact and internal pressure. Archives of Civil and Mechanical Engineering. 2018;18:1715-28.

[24] Tekieli M, De Santis S, de Felice G, Kwiecień A, Roscini F. Application of Digital Image Correlation to composite reinforcements testing. Composite Structures. 2017; $160: 670-88$.

[25] Seif MA, Khashaba UA, Rojas-Oviedo R. Measuring delamination in carbon/epoxy composites using a shadow moiré laser based imaging technique. Composite structures. 2007;79:113-8.

[26] Callaway P, Gilbert M, Smith CC. Influence of backfill on the capacity of masonry arch bridges. Proceedings of the Institution of Civil Engineers: Bridge Engineering: ICE Publishing; 2012. p. 147-57.

[27] Sánchez-Aparicio L, Villarino A, García-Gago J, González-Aguilera D. Photogrammetric, geometrical, and numerical strategies to evaluate initial and current conditions in historical constructions: A test case in the church of San Lorenzo (Zamora, Spain). Remote Sensing. 2016;8:60.

[28] Pan B. Digital image correlation for surface deformation measurement: historical developments, recent advances and future goals. Measurement Science and Technology. 2018;29:082001.

[29] Daghash SM, Ozbulut OE. Flexural performance evaluation of NSM basalt FRPstrengthened concrete beams using digital image correlation system. Composite Structures. 2017; 176:748-56.

[30] Unlusoy C, Melenka GW. Flexural testing of cellulose fiber braided composites using three dimensional digital image correlation. Composite Structures. 2019;230:111538.

[31] He T, Liu L, Makeev A. Uncertainty analysis in composite material properties characterization using digital image correlation and finite element model updating. Composite Structures. 2018;184:337-51.

[32] Yu L, Pan B. Experimental study of tensile properties and deformation evolutions of $2 \mathrm{D}$ and $2.5 \mathrm{D}$ woven $\mathrm{SiO} 2 \mathrm{f} / \mathrm{SiO} 2$ composites using single-camera stereo-digital image correlation. Composite Structures. 2018;200:589-98.

[33] Kowalczyk P. Identification of mechanical parameters of composites in tensile tests using mixed numerical-experimental method. Measurement. 2019;135:131-7.

[34] Ahn J, He E, Chen L, Dear J, Shao Z, Davies C. In-situ micro-tensile testing of AA2024-T3 fibre laser welds with digital image correlation as a function of welding speed. International Journal of Lightweight Materials and Manufacture. 2018;1:179-88. [35] Xu D, Cerbu C, Wang H, Rosca IC. Analysis of the hybrid composite materials reinforced with natural fibers considering digital image correlation (DIC) measurements. Mechanics of Materials. 2019;135:46-56.

[36] Garcia-Martin R, Bautista-De Castro Á, Sánchez-Aparicio LJ, Fueyo JG, GonzalezAguilera D. Combining digital image correlation and probabilistic approaches for the reliability analysis of composite pressure vessels. Archives of Civil and Mechanical Engineering. 2019;19:224-39. 
612 [37] das Neves Carneiro G, António CC. Global optimal reliability index of implicit

613 composite laminate structures by evolutionary algorithms. Structural Safety. 2019;79:54-

61465 .

615 [38] Rafiee R, Torabi MA. Stochastic prediction of burst pressure in composite pressure

616 vessels. Composite Structures. 2018;185:573-83.

617 [39] Rafiee R, Reshadi F, Eidi S. Stochastic analysis of functional failure pressures in

618 glass fiber reinforced polyester pipes. Materials \& Design. 2015;67:422-7.

619 [40] Rafiee R. Stochastic fatigue analysis of glass fiber reinforced polymer pipes. 620 Composite Structures. 2017;167:96-102.

621 [41] Tomar SS, Zafar S, Talha M, Gao W, Hui D. State of the art of composite structures 622 in non-deterministic framework: a review. Thin-Walled Structures. 2018;132:700-16.

623 [42] Dey S, Mukhopadhyay T, Adhikari S. Metamodel based high-fidelity stochastic 624 analysis of composite laminates: A concise review with critical comparative assessment. 625 Composite Structures. 2017;171:227-50.

626 [43] ISO E. Plastics-determination of tensile properties. 1997.

627 [44] Toutanji H, Dempsey S. Stress modeling of pipelines strengthened with advanced 628 composites materials. Thin-Walled Structures. 2001;39:153-65.

629 [45] ISO E. 527-4. Plastics-determination of tensile properties-part 4: test conditions for 630 isotropic and orthotropic fibre-reinforced plastic composites. International Organization 631 for Standardization (ISO), Geneva, Switzerland. 1997.

632 [46] Sutton MA, Orteu JJ, Schreier H. Image correlation for shape, motion and 633 deformation measurements: basic concepts, theory and applications: Springer Science \& 634 Business Media; 2009.

635 [47] Pan B, Qian K, Xie H, Asundi A. Two-dimensional digital image correlation for in636 plane displacement and strain measurement: a review. Measurement science and 637 technology. 2009;20:062001.

638 [48] Dong Y, Pan B. A review of speckle pattern fabrication and assessment for digital 639 image correlation. Experimental Mechanics. 2017;57:1161-81.

640 [49] Vo MN, Wang Z, Luu L, Ma J. Advanced geometric camera calibration for machine 641 vision. Optical Engineering. 2011;50:110503.

642 [50] Martins LA, Bastian FL, Netto TA. Structural and functional failure pressure of 643 filament wound composite tubes. Materials \& Design (1980-2015). 2012;36:779-87.

644 [51] Tsai SW, Wu EM. A general theory of strength for anisotropic materials. Journal of 645 composite materials. 1971;5:58-80.

646 [52] Novak L, Novak D. Polynomial chaos expansion for surrogate modelling: Theory 647 and software. Beton-und Stahlbetonbau. 2018;113:27-32.

648 [53] Bautista-De Castro Á, Sánchez-Aparicio LJ, Carrasco-García P, Ramos LF, 649 González-Aguilera D. A multidisciplinary approach to calibrating advanced numerical 650 simulations of masonry arch bridges. Mechanical Systems and Signal Processing. $6512019 ; 129: 337-65$.

652 [54] Wiener N. The homogeneous chaos. American Journal of Mathematics. 653 1938;60:897-936.

654 [55] Blatman G, Sudret B. Adaptive sparse polynomial chaos expansion based on least 655 angle regression. Journal of Computational Physics. 2011;230:2345-67.

656 [56] Efron B, Hastie T, Johnstone I, Tibshirani R. Least angle regression. The Annals of 657 statistics. 2004;32:407-99.

658 [57] Deman G, Konakli K, Sudret B, Kerrou J, Perrochet P, Benabderrahmane H. Using 659 sparse polynomial chaos expansions for the global sensitivity analysis of groundwater 660 lifetime expectancy in a multi-layered hydrogeological model. Reliability Engineering \& 661 System Safety. 2016;147:156-69. 
662 [58] Lecompte D, Sol H, Vantomme J, Habraken A. Analysis of speckle patterns for 663 deformation measurements by digital image correlation. International Society for Optics 664 and Photonics; 2006. p. 63410E.

665 [59] Pan B, Lu Z, Xie H. Mean intensity gradient: an effective global parameter for 666 quality assessment of the speckle patterns used in digital image correlation. Optics and 667 Lasers in Engineering. 2010;48:469-77.

668 [60] Ab Ghani AF, Ali MB, DharMalingam S, Mahmud J. Digital image correlation 669 (DIC) technique in measuring strain using opensource platform Ncorr. Journal of 670 Advanced Research in Applied Mechanics. 2016;26:10-21.

671 [61] Sasikumar P, Suresh R, Vijayaghosh PK, Gupta S. Experimental characterisation of 672 random field models for CFRP composite panels. Composite Structures. 2015;120:45167371.

674 [62] D'Agostino RB. Goodness-of-fit-techniques: CRC press; 1986.

675 [63] Liu H. Pipeline engineering: CRC Press; 2003.

676 [64] Marelli S, Sudret B. UQLab: A framework for uncertainty quantification in Matlab.

677 Vulnerability, Uncertainty, and Risk: Quantification, Mitigation, and Management2014. 678 p. 2554-63.

679 [65] McKay MD, Beckman RJ, Conover WJ. Comparison of three methods for selecting 680 values of input variables in the analysis of output from a computer code. Technometrics. $681 \quad 1979 ; 21: 239-45$.

682 [66] Goldberg D. 'genetic algorithms in search, optimization \& machine learning', 683 addison-wesley publishing company, inc., 1989. 1989.

684 [67] Mitchell M. An introduction to genetic algorithms: MIT press; 1998. 